\title{
Meeting Report: \\ AAPS Workshop on Inhalation Product \\ Biopharmaceutical Classification System Development: Challenges and Opportunities
}

Vivian A. Gray

Dissolution Technologies, Hockessin, DE, USA

e-mail: vagray@rcn.com

\section{T} he AAPS Workshop on Inhalation Product Biopharmaceutical Classification System Development was held in Baltimore, MD, on March 16-17, 2015. The workshop was sponsored by AAPS, USP, and FDA. The goal of the workshop was to determine whether an inhalation product biopharmaceutics classification system (BCS) based on fundamental physicochemical properties of physiologic importance could be developed for inhalation drug products. Members of the Program Committee were Jeffrey Breit (Bend Research and Capsugel), Sai Chamarthy (Merck), Jayne Hastedt (JDP Pharma Consulting, LLC), Bill Doub (FDA), Bill Schachmer (Lupin), and Tony Hickey (RTI International).

Opening remarks were given by Jayne Hastedt, who reiterated the goals of the workshop, provided a brief outline of the history of BCS for oral dosage forms, and applied the term iBCS for a BCS for inhaled products. She told the participants that after the formal presentations there would be Breakout Sessions on the following topics: Dose Number and iBCS, Absorption Number and iBCS, and Dissolution Number and iBCS. A white paper on the key learnings from the workshop will be published.

The first talk, given by Gordon Amidon (University of Michigan), was titled "BCS: Scientific Base and Extensions to Inhalation." He began by working through the steps in oral BCS development and identifying the key concepts. He felt that by considering all of the key concepts a unifying scientific principle would emerge from the two dosage forms. In approaching iBCS, a description of the unique physiology and biology of the route of administration is necessary, including a discussion of mass transport analysis and release mechanisms. The basis for oral BCS, which he referred to as giBCS, is that the drug is absorbed through the intestinal membrane at a rate that is proportional to the concentration at the membrane surface. The dissolution of the drug product in vivo determines the membrane surface concentration of the drug. For iBCS, the basis will include such factors as time-dependent surface area, direct contact of active ingredient and excipients with absorbing surface, time-dependent physical-chemical changes, and local activity. There is a need to identify what variables are more important, with local-versus-systemic absorption heading the list along with local pharmacology.

Then Peter Mauser (Merck) spoke on "The Target Organ: Lung Anatomy and Physiology." He began with an introduction to the lungs and the physiological responses to drug inhalation, emphasizing the anatomical and physiological relationship of the lung, airway size, and deposition of particles. He discussed the physiological considerations affecting particle deposition in the lungs and described the lung as a defensive barrier to particulate inhalation. Particle dissolution and absorption was described in the two zones of the lung, central and respiratory. He then gave a comparison of physiological aspects of inhaled and oral drug delivery. A major difference between oral and inhalation routes of delivery is the timing of the PK. With inhaled absorption, the PK is rapid, there is no first pass, and therefore major enzymatic attack from the liver is avoided. If properly engineered, the lung provides a superior route of delivery for compounds not amenable by oral delivery or requiring rapid onset of action.

After the break, Claus-Michael Lehr (Helmholtz Institute for Pharmaceutical Research, Saarland University) discussed "Lung Transport and Permeability: The Fate of Inhaled Drugs." He first claimed that pulmonary drug delivery is a promising new era for nanomedicine and spoke of a new paradigm with emphasis on controlling the fate of particles after deposition. Examples of this idea are improving absorption across the air-blood barrier, controlling and avoiding pulmonary clearance, and cell-specific targeting. Dissolution testing of inhalation products was described in historical terms where in 2008, an ad hoc advisory panel of the USP stated there was no need for dissolution testing of the products presently on 
the market (1). In 2012, an International Pharmaceutical Aerosol Consortium on Regulation and Science review stated that dissolution testing is a valuable tool in the development of inhaled dosage forms. However, there is currently no dissolution method that can be used as a standard. From 2000 to 2013 several dissolution methods that used a modified twin stage impinger (2), flowthrough cell (3-5), USP Apparatus $2(4-6)$, Franz cell $(4,5)$, and Transwell $(7,8)$ were in the literature. He concluded with recent applications of inhalation nanocarriers for advanced pulmonary drug delivery.

The afternoon session began with Andrew Clark (Respira Therapeutics) giving a presentation on "Fluid Mechanics and Pulmonary Drug Deposition-Lung Dose." He began with a review of the airway structure and biology of the lung. He remarked that extreme variations in regional depositions can affect PK and that regional deposition still has to be calculated using numerical models. Central deposition appears to be faster, possibly due to mucociliary clearance. PK differences are hard to determine with polydispersed aerosols. He pointed out that particle size, flow rate, and regional deposition produce local variation in drug concentration; however, intersubject variability is also high. Clark also said that IVIVC for total lung deposition using oropharyngeal models appears feasible. He concluded by posing the question, how does the interplay of deposition, solubility, and dissolution really influence PK? and stated that PK does not necessarily predict efficacy or duration of action. The next speaker, Jeffry Weers (Novartis), spoke on "Particle Dissolution in the Lung: Basic Principles." He first asked if particle dissolution would be a critical quality attribute (CQA) in the Quality by Design ( $Q b D$ ) of orally inhaled products (OIP). He mentioned that there are currently no regulatory requirements or pharmaceutical techniques for dissolution testing of OIPs (8). He quoted the 2008 publication that states that there was no compelling evidence that dissolution was "kinetically and/ or clinically critical for currently approved" OIPs (1). The 2012 consortium publication (8) commented that "there is still insufficient knowledge for translating the dissolution data into statements about quality, performance, safety, or efficacy of OIPs in general." The publication also stated that a relationship between clinical data and dissolution has yet to be established. For particle dissolution in the lung, the dissolution rate depends critically on solubility and surface area, making it most important for drugs that are considered "insoluble." He described how the surfaces for dissolution vary throughout the lung, comparing the lung to a bucket and the gastrointestinal tract to a tube (9). Weers gave representative dose numbers $\left(D_{0}\right)$ for various respiratory therapeutics in the conducting airways and said that dissolution number $\left(D_{n}\right)$ is expected to be much greater for OIP due to the small particle size. He described the dissolution of indomethacin nanoparticles (10). He concluded by questioning the importance of dissolution in terms of clinical performance of OIPs, stating that absorption and metabolism require the drug to be in solution. Dissolution-limited absorption, until recently, has been important for only a small subset of APIs. In vivo and in vitro would be difficult due to the complexity of particle deposition and lung clearance. Kinetics for particle dissolution may be very different from the kinetics of systemic absorption. Finally, particle dissolution testing may be most useful for early development of pulmonary controlled-release formulations.

Jason McConville (University of New Mexico) spoke next on "Particle Dissolution in the Lung: Analytical Testing." He began his talk by comparing drug dissolution in the gastrointestinal tract (GIT) with that in the lung. For both, the surface area for drug absorption is important. In the GIT, disintegration is critical for the drug to dissolve and be absorbed, whereas in the lung, there is a large surface area for absorption and apparently, absorption of powders happens quickly. The GIT has a high liquid volume while the lung has a low volume. The $\mathrm{pH}$ range for the GIT is $1-8$, whereas the lung $\mathrm{pH}$ is around $6-7$. There are high hydrodynamic conditions in the GIT. In the lung, the hydrodynamic conditions are low and there are also macrophage interactions to consider. He then discussed the in vitro dissolution conditions for the GIT, including sink conditions, of volumes from 150 to $900 \mathrm{~mL}$ using buffered media. The in vitro experimental conditions for inhaled products include dispersed particles with a large surface area for dissolution using media of a fixed $\mathrm{pH}$, with simulated lung fluid as an option; sink conditions are an uncertainty. He pointed out that dispersed particles offer a challenge to in vitro dissolution testing and discussed various instrumental approaches: the Modified Type 1 Apparatus (11), Modified HPLC pump system (2), and Modified TSI (12). He went on to describe an electrostatic dispersion method and an aerodynamic separation method using a dissolution membrane diskette (13). He discussed the lung surfactant and presented a simulated lung fluid preparation (2). He concluded with examples of the utility of a membrane diskette in generating data where the Higuchi model may be applied.

Hiro Sakagami (Virginia Commonwealth University) presented the last talk of the session, "PK/PD Modeling for Lung Delivery." This talk began with a discussion of how, with accurate dose measures, human PK/PD data 
likely enable the assessment of systemic efficacy and safety of inhaled products. However, there are conceptual complexities and quantitative difficulty with systemic or local delivery. The assessment of local safety and efficacy remains theoretical, as local lung PK and appropriate PD measures are missing. He explored animal PK/PD for lung delivery to predict human PK. Animal studies enable the assessment of drug/formulation-dependent PK/PD, while their delivery/deposition-dependent PK/PD is likely not relevant to humans. For human PK/PD, he explained the complexity and interplay of dose, regional deposition, and difficulty of PD measures. He concluded with a description of a theoretical model for prediction that would include inhaled and swallowed fraction, drug dissolution, mucociliary clearance, lung absorption, and receptor binding affinity.

The second day's session began with three case studies. Per Bächman (AstraZeneca) presented "Local Lung Delivery: Small Molecule Case Study Example \#1." In this example, the compound studied was an inhaled selective glucocorticoid receptor modulator (SGRM) with low water solubility. From the data set, he relayed the observed impact of deposition, dissolution, and mucociliary clearance on systemic exposure. The study showed a link between observed systemic exposure and simulated local and systemic exposure. He closed with his thoughts on a classification system/predictive model for locally acting inhaled medicines, which were essentially that a mechanistic model has the best chance of predicting clinical exposure.

The second case study was presented by Günther Hochhaus (University of Florida) on "iBCS Case Study 2: Small Molecular Weight Compounds for Pulmonary Delivery." He began by relating the differences between oral and inhalation delivery, then posed the questions, what test should we use? and how could we classify inhaled oral compounds (IOCS) without an iBCS? He pointed out that the IOCs are both a device and a formulation, whereas for oral drug products there is just a formulation. This is a significant difference between two products when considering a biowaiver. The lung regional depositions are another consideration unique to IOCs. He discussed twelve active ingredients used in IOCs. He concluded that pursuing iBCS is worthwhile, but the tools need to be improved. Those tools include the dissolution test, permeability mechanism, and the differences between central and peripheral lungs.

The last case study, presented by Ralph Niven (Novartis), was "The Lungs, Particles, Clearance, and Atypical Molecules." He explained that theoretically, high solubility is required for a particle to dissolve in the alveolar fluid; however, this does not fit with the rate and extent of systemic availability of many molecules via the lungs. The contributing factors to this apparent high solubility are the nature of the particles, the surfactants in the lung environment, alveolar movement, and the disease state. He also discussed the responsiveness of the lung environment, including fluid exchange and macrophage activity. He then addressed the subject of biologics, wondering if any characteristics of biologics are predictive of bioavailability. He went on to explain that the literature data set is too small and there are numerous confounders, with not much that is systematic or standardized. There are few in vivo techniques, beyond a PK evaluation of systemic data, to gauge drug/biologic behavior in the lungs. He asked if a case could be made for an "internal standard." He concluded that there was no robust methodological or design consistency with in vivo data sets compiled from the literature rendering it difficult to draw robust conclusions about the post-deposition behavior of biologics and many small molecules. These case studies were followed by a roundtable discussion, "Small Molecule Considerations for BCS Classification," moderated by Bill Schachtner (Lupin).

The afternoon was spent in three Breakout sessions, Dose Number and iBCS, Absorption Number and iBCS, and Dissolution Number and iBCS, which were followed by summaries from the moderators of the breakout sessions. The conference was brought to a close by Jayne Hastedt, who summarized the proceedings, discussed the next steps to evaluate the classification approaches, and told the audience that there would be a publication summarizing the workshop outcomes and findings. She thanked the planning committee and the participants for a very productive workshop.

\section{REFERENCES}

1. Gray, V. A.; Hickey, A. J.; Balmer, P.; Davies, N. M.; Dunbar, C.; Foster, T. S.; Olsson, B. L.; Sakagami, M.; Shah, V. P.; Smurthwaite, M. J.; Veranth, J. M.; Zaidi, $K$. The Inhalation Ad Hoc Advisory Panel for the USP performance tests of inhalation dosage forms. Pharm. Forum 2008, 34 (4), 1068-1074.

2. Davies, N. M.; Feddah, M. R. A novel method for assessing dissolution of aerosol inhaler products. Int. J. Pharm. 2003, 255 (1-2), 175-187. DOI: 10.1016/ S0378-5173(03)00091-7.

3. Salama, R. O.; Traini, D.; Chan, H.-K.; Young, P. M. Preparation and characterisation of controlled release co-spray dried drug-polymer microparticles for inhalation 2: Evaluation of in vitro release profiling methodologies for controlled release respiratory aerosols. Eur. J. Pharm. Biopharm. 2008, 70 (1), 145152. DOI: 10.1016/j.ejpb.2008.04.009. 
4. Son Y. J.; Horng M.; Copley M.; McConville J. T. Optimization of an In Vitro Dissolution Test Method for Inhalation Formulations. Dissolution Technol. 2010, 17 (2), 6-13. DOI: 10.14227/DT170210P6.

5. May, S.; Jensen, B.; Wolkenhauer, M.; Schneider, M.; Lehr, C. M. Dissolution Techniques for In Vitro Testing of Dry Powders for Inhalation. Pharm. Res. 2012, 29 (8), 2157-2166. DOI: 10.1007/s11095-012-0744-2.

6. Arora, D.; Shah, K. A.; Halquist, M. S.; Sakagami, M. In Vitro Aqueous Fluid-Capacity-Limited Dissolution Testing of Respirable Aerosol Drug Particles Generated from Inhaler Products. Pharm. Res. 2010, 27 (5), 786795. DOI: 10.1007/s11095-010-0070-5.

7. Bhagwat, S.; Rohrschneider, M.; Alfadehl, S.; Hochhaus, G. Development of An In-Vitro Test Method For Dissolution Of Inhaled Corticosteroids. International Society for Aerosols in Medicine Conference, University of North Carolina, Chapel Hill, NC, Aug 6-10, 2013.

8. Riley, T.; Christopher, D.; Arp, J.; Casazza, A.; Colombani, A.; Cooper, A.; Dey, M.; Maas, J.; Mitchell, J.; Reiners, M.; Sigari, N.; Tougas, T.; Lyapustina, S. Challenges with Developing In Vitro Dissolution Tests for Orally Inhaled Products (OIPs). AAPS PharmSciTech 2012, 13 (3),
978-989. DOI: 10.1208/s12249-012-9822-3.

9. Halstedt, J. E. The Lung as a Dissolution Vessel? Inhalation 2014, 8 (6), 18-22.

10. Laaksonen, T.; Liu, P.; Rahikkala, A.; Peltonen, L.; Kauppinen, E. I.; Hirvonen, J. Intact Nanoparticulate Indomethacin in Fast-Dissolving Carrier Particles by Combined Wet Milling and Aerosol Flow Reactor Methods. Pharm. Res. 2011, 28 (10), 2403-2411. DOI: 10.1007/s11095-011-0456-z.

11. Jaspart, S.; Bertholet, P.; Piel, G.; Dogné, J.-M.; Delattre, L.; Evrard, B. Solid lipid microparticles as a sustained release system for pulmonary drug delivery. Eur. J. Pharm. Biopharm. 2007, 65 (1), 47-56. DOI: 10.1016/j.ejpb.2006.07.006.

12. McConville, J. T.; Patel, N.; Ditchburn, N.; Tobyn, M. J.; Staniforth, J. N.; Woodcock, P. Use of a Novel Modified TSI for the Evaluation Of Controlled-Release Aerosol Formulations. Drug. Dev. Ind. Pharm. 2000, 26 (11), 1191-1198. DOI: 10.1081/DDC-100100991.

13. Son, Y.-J.; McConville, J. T. Development of a standardized dissolution test method for inhaled pharmaceutical formulations. Int. J. Pharm. 2009, 382 (1-2), 15-22. DOI:10.1016/J.IJPHARM.2009.07.034. 